\title{
Real-time Evaluation Mechanism Based on Double Evidence Classification of User Behavior
}

\author{
Jiale Zhang ${ }^{1}$, Guiling Zhang ${ }^{1, *}$ and Xiufang Zhang ${ }^{2}$ \\ ${ }^{1}$ School of Computer Science \& Software Engineering, Tianjin Polytechnic \\ University, Tianjin, 300387, China \\ ${ }^{2}$ Institute of Mathematics \& Information Science, Langfang Teachers University, \\ Langfang, 0650007, China \\ *glzhang808@sohu.com
}

\begin{abstract}
With the development of trusted network, the research of trusted evaluation mechanism of user behavior is a hotpot in the network security. In order to solve the problems of subjectivity, limitations and static in traditional trusted network user behavior evaluation models, we have to find a real-time and dynamic evaluation method for user behavior. In this paper, the authors construct a real-time evaluation mechanism based on double evidence classification of user behavior (DEC-UB). The evaluation mechanism includes the process classification and characteristic classification of user behavior evidence, which makes the user behavior evidence of any time can be directly involved in the trust evaluation, and the evaluation result is more comprehensive and accurate. Simulation experiments have evaluated the three kinds of user behaviors based on the DEC-UB, and compared them with the other two kinds of trust evaluation methods of user behavior, the results show that the proposed methods can evaluate the user's behavior comprehensively, accurately and dynamically in complex network environments, and the results are more realistic.
\end{abstract}

Keywords: Trusted network, user behavior, trusted evaluation, evidence classification, $D E C-U B$

\section{Introduction}

Following the development of network technology and applications, the traditional network application model can't meet the user's network needs. Distributed systems (such as cloud computing, grid computing, $\mathrm{P} 2 \mathrm{P}$, an ad hoc networks and e-commerce, etc.) is rapidly becoming the mainstream in the development of networks. Users can get more opportunities to share resources and collaborate interactively in this network environment. A number of user behaviors will endanger the running state of the network by different degrees, and even affect the normal interaction of other users, such as users of illegal access, abuse of resources and malicious attack behavior, will lead to network congestion, network load, abnormal nodes or even network paralysis. Therefore, the research of Internet users trust is of great significance to build a trusted network environment.

Internet users' trust includes not only the user's identity trust, but also the user's behavior trust. The user's identity trust refers to how the end-user's identity can be accurately identified, not posing as someone else. User behavior trust refers to whether the end-user's behavior can be assessed, is predictable, has manageable network devices and whether the data will result in damage or destruction. The traditional authorization and authentication superficially solves the problem of user identity trust, but does not solve the problem of user behavior trust of users. This is obviously unreasonable because the user is not only able to create and store important data sources, but also the vast majority of attacks are also initiated from the users. Therefore, research on the user 
behavior trust is needed to clear the need for trusted network firstly, and the trusted network must study the behavior trust of users on the basis of traditional user identity trust. At the same time, as the user behavior trust is not only more detailed than the control granularity of the identity trust, but also is a computation dynamic trust model for user authorization, it will strengthen the user's trust as one of the important components of the trusted network.

In this paper, the proposed evaluation mechanism is based on DEC-UB and can doubly classify the user behavior evidence, whilst the double slide window mechanism offers real-time control of the trust evaluation process. This makes the user behavior evidence can be directly involved in the trust evaluation at any time, and the evaluation result is more comprehensive and accurate. What's more, the trust values are calculated using a simple iterative update method, which makes the evaluation result more quantitative.

\section{Stage of the Research}

At this stage, the researches on user behavior trust is mainly reflected in the following three aspects:

(1) Firstly, the researches on user behavior trust are focused on how to build trust models of user behavior in network and the network access control model based on user behavior trust. The paper [1] proposed a trust management architecture that can evolve and maintain the behavior trust based on user's historical behavior as well as correspondent trust level; The paper [2] detail a procedure for computing reputationbased trust assessments relying on user demographics and from provenance, and then proposed a procedure for computing trust values based on provenance information, represented using w3c prov model; The paper [3] built a dynamic trust model based on indirect trust relationship, this method take advantage of keynote to build the dynamic trust model, in which used an indirect trust algorithm to calculate the trust value of indirect trust relationship; The paper [4] provided a security framework suitable for dependent people by using the hug and various contextual data issued from the sensors deployed in smart environments; The paper [5] improved the calculation method of indirect credibility by thinking the existing problems of the user behavior trust evaluation method in the subjective weight and dynamic adaptability; The paper [6] proposed a computational dynamic trust model for user authorization, rooted in findings from social science, unlikely most existing computational trust models, this model distinguishes trusting belief in in tegrity from that in competence in different contexts and accounts for subjectivity in the evaluation of a particular trustee by different trusters; The paper [7] recognized reputation, recommendation and rating systems as online trust representatives and explores the biased behavior resulting from users' perception of those systems, and then built a framework for addressing some of the issues attributed to users' biased behavior.

(2) With the development of cloud computing, the hot spot of research is now moving into the cloud trust model of user behavior, and the evaluation mechanism based on cloud trust model. Such as the fuzzy logic technique [8] based on cloud computing, and the mutual trust based access control model [9] combined with trust management. What's more, the paper [10] proposed a dynamic trust evaluate method to deal with cloud user's behavior by using entropy method and AHP, and the paper [11] proposed a new method for user behavior trust evaluation that considers user's identification code and mac address to prevent the registration of members with multiple user names and makes available the information of user behavior trust in other cloud computing environments. 
(3) Research applications trust evaluation on the terminal user behavior, especially research for mobile applications has made important achievement. The paper [12] presented the design and implementation of a trust-behavior based reputation system for mobile applications based on android system; The paper [13] presented a trust-behavior-based reputation and recommendation system for mobile applications; The paper [14] have put forward the trust management approach by analyzing user behavioral patterns for reliable mobile cloud computing, and suggested a method to quantify a one-dimensional trusting relation based on the analysis of telephone call data form mobile devices; The paper [15] proposed a prediction method of mobile user preferences based on trust and link prediction by analyzing mobile user behaviors, the experimental results show that the method can obtain more accurate mobile user preferences compared with traditional collaborative filtering, and it can solve the sparsity issuer to some extent.

The existing results have mainly studied the static evaluation of user behavior and other applications. However the research on real-time and dynamic evaluation methods of user behavior is not comprehensive, and this is the key point of this paper.

\section{Real-time Evaluation Mechanism}

\subsection{Relevant Definitions}

Definition. 1. User Behavior Trust (UBT): User behavior trust refers to the interaction between two or more users, according to the behavior of users in the process of interaction to make evaluation.

Definition. 2. User Behavioral Evidence (UBE): User behavior evidence refers to the basic values that can be used to quantitatively assess the overall behavior of the user based on the software and hardware testing.

Definition. 3. User Behavior Trust Value (UBTV): User behavior trust value with specific values to characterize the credible degree of the user behavior, denoted by $\mathrm{T}$ $(T \in[0,1])$, when the higher the value, the greater the credible degree.

Definition. 4. Evidence of Membership [16]: For the degree of membership fuzzy concept of "excellent", exported by Chad membership degree equation used to characterize the evidence.

a. For larger and more superior types of evidence:

$$
g=\frac{e-\operatorname{Inf}(e)}{\operatorname{Sup}(e)-\operatorname{Inf}(e)}
$$

b. For smaller the more superior types of evidence:

$$
g=\frac{\operatorname{Sup}(e)-e}{\operatorname{Sup}(e)-\operatorname{Inf}(e)}
$$

Wherein $\mathrm{g}$ is evidence of membership degree, e is the result of evidence obtained by numerical calculation, $\operatorname{Sup}(\mathrm{e})$ and $\operatorname{Inf}(\mathrm{e})$ respectively represent the evidence value of the upper and lower bounds.

Definition. 5. Successful Transaction Rate (STR): Successful transaction rate refers to the ratio of the number of successful network user interactions that occur throughout all transactions in the account. STR indicators are used to measure the accuracy of a credible assessment of policies. In a dynamic network environment, the higher STR is, the more accurate the description of credible assessment of policies, and the stronger the dynamic adaptability. 


\subsection{Evaluation Model of User Behavior Trust}

Evaluation model of user behavior trust is a hot issue in the trusted network, the traditional user behavior trust evaluation methods were belongs to the static evaluation mechanism. However, the evaluation results become invalid extremely easily because terminal platform environments and the user behaviors changing in the actual network environment, which lacks of a real-time and dynamic evaluation method for user behavior. In this research, we proposed an evaluation model based on double evidence classification of user behavior (DEC-UB). The flow chart is as shown in Figure 1.

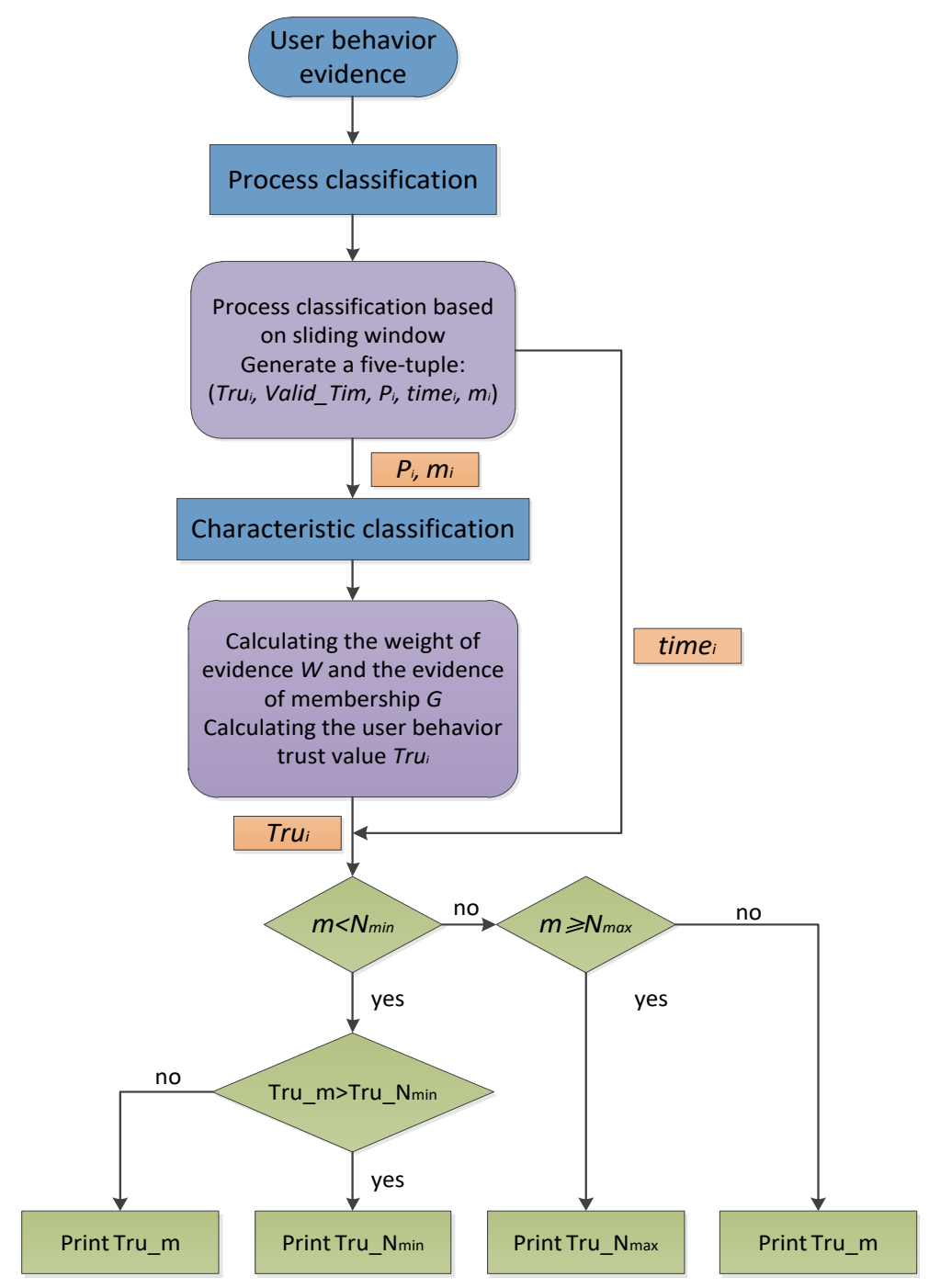

Figure 1. The Flow Chart based on DEC-UB

Firstly, use the double slide window to classify the user behavior evidence based on process, and generate a five-tuple (Tru , Valid_Tim, $P_{i}$, time $\left.e_{i}, m_{i}\right)$. The user behavior evidence is divided into different time spans of the evidence set $\mathrm{Pi}$, and then classified so that each time span of effective behavioral evidence sets based on different characteristics. This is so as to calculate the user behavior trust value, and iterative updating trust value through the double sliding window mechanism. Finally, according to the actual user trust records and window size of the comparison results, we can determine the final trust value. 


\subsection{Process Classification Method for User Behavior Evidence (UBE)}

\subsubsection{Process Classification Based on DSW}

The classification method based on the double sliding window of the user behavior \left. evidence process consists of a five-tuple (Tru ${\text {, Valid_Tim, } P_{i} \text {, time }}_{i}, m_{i}\right)$.

(1) User behavior trust value of the trust validity period $T r u_{i}$ is used to represent the user behavior of credibility assessment.

(2) Trust effective time span Valid_Tim is used to determine whether the user behavior trust records expired.

(3) Evidence sets of the trust validity period $P_{i}$.

(4) Actual access time of the trust validity period time $_{i}$ is used to record the N-th trust value of time, reflect the different time period of the performance evaluation results are different.

(5) User actual access times of trust validity period $m_{i}$ is used to evaluate the user actual behavior trust value in the window.

Due to the final user behavior trust evaluation mechanism being based on the trust of the user access to long-term dynamic updating and comprehensive evaluation results, the user evaluation process can be viewed as a double sliding window model as shown in Figure 2, where two windows share a window trailing edge. The left window (trust established window) is used to control the sliding window of trust established, and the right window (extensible window) is used to control the sliding window extensibility.

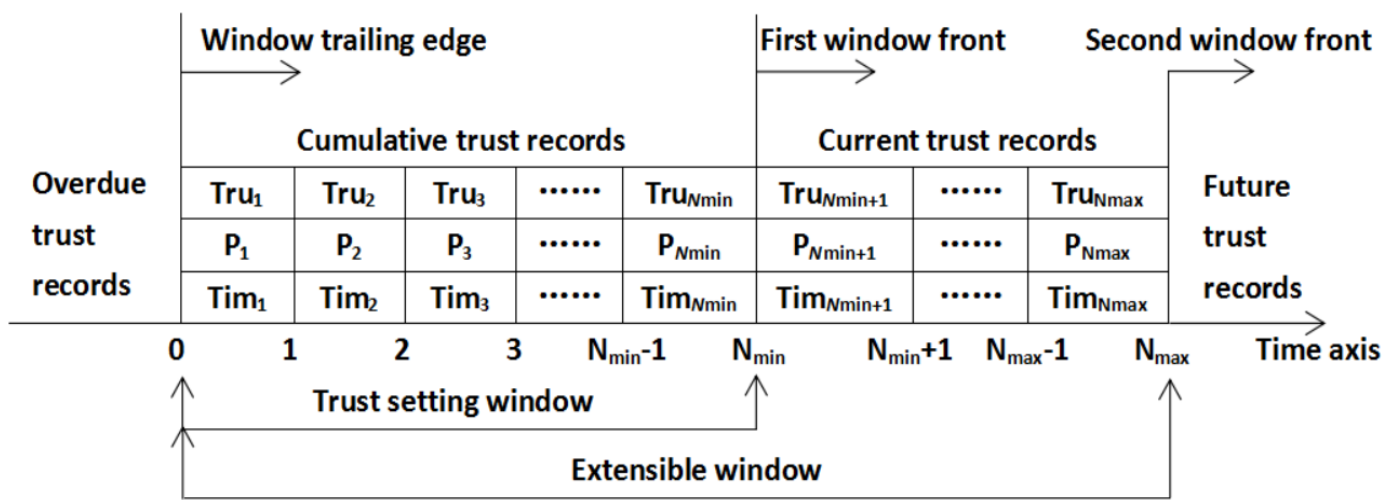

Figure 2. Process Classification for User Behavior

When the user does not visit for a long time, a number of trust records will be far away from the current time, and gradually become outdated trust records (time new $^{-}$ time $_{i}>$ Valid_Tim). The value of the old trust record no longer retains the original value of trust records, but instead is replaced by the value of the relatively low unknown trust records. Replacement strategy, as used in this paper, is the furthest time replacement policy. Time is the same as the leftmost valid record time, and the tag is set to stranger so the trust record is sorted by time, which is replaced by the record on the left side of the window.

\subsubsection{Determination of Time Factor}

The basic idea of the user behavior trust value update mechanism is that the more recent behavior in the comprehensive assessment of the proportion of the greater, in the double slide window, it means that the closer to the left side, the smaller the proportion in the trust evaluation, and the closer to the right side, the larger the proportion in the trust 
evaluation. In this paper, we proposing the Time Factor to measure this proportion. The time factor $d$ was computed as follow:

$$
d_{s}=\frac{\left(\text { time }_{s}-\text { time }_{1}\right)}{\sum_{s=1}^{n}\left(\text { time }_{s}-\text { time }_{1}\right)}
$$

\subsection{Characteristic Classification Method for UBE}

\subsubsection{Characteristic Classification Method}

After the process of classification for user behavioral evidence, we need to quantitatively evaluate the user behavior trust based on a per time span. Because the user's behavior evidence data set is very large and complex, and it is difficult to carry out data processing, we should classify the evidence of user behavior based on different characteristics, and divide the user behavior which includes evidence factors into three different layers. Respectively, for the Target layer, Criterion layer (Characteristic layer) and Measure layer (Evidence layer). Thereby we can create a user behavior evaluation model as shown in Figure 3.

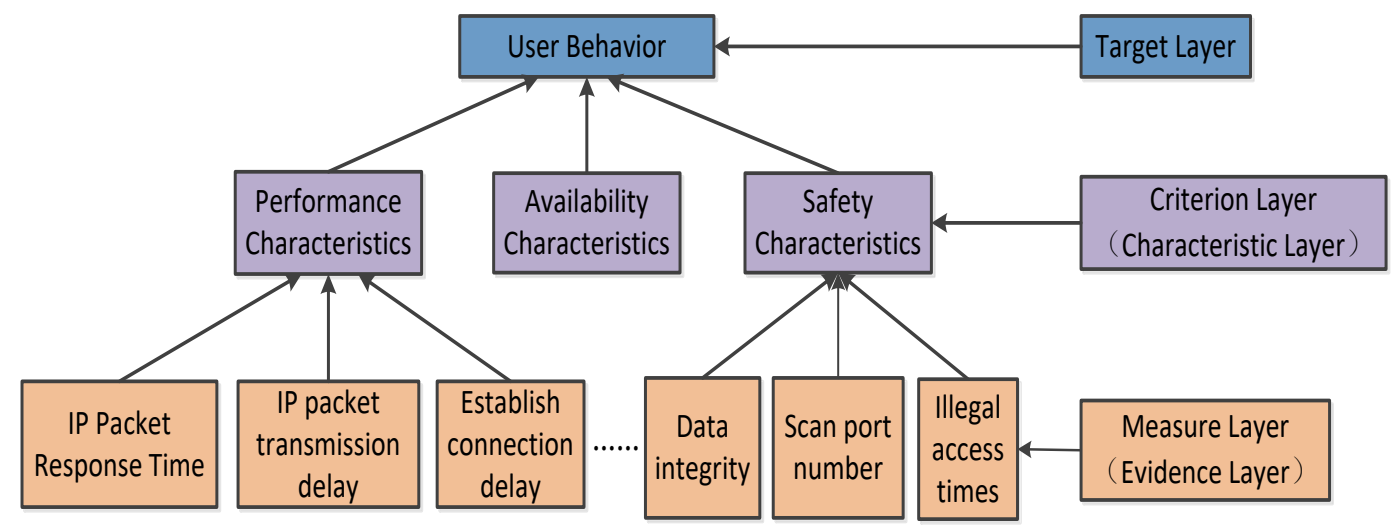

Figure 3. Characteristic Classification for User Behavior

\subsubsection{Calculate the Weight of UBE}

Suppose that a user behaviors evidence is associated with security features with $n$, respectively, for $P_{1}, P_{2}, \ldots \ldots, P_{n}$, and according to the relative importance of this evidence in security features, we can get the $\mathrm{n}$ order judgment matrix as follow:

$$
\begin{gathered}
P_{1} P_{2} \\
P_{i j}= \\
P_{1}\left[\begin{array}{cccc}
p_{11} & p_{12} & \ldots & P_{1 n} \\
P_{2} & \ldots \\
p_{21} & p_{22} & \ldots & p_{2 n} \\
\ldots & \ldots & \ldots & \ldots \\
P_{n 1} & p_{n 2} & \ldots & p_{n n}
\end{array}\right]
\end{gathered}
$$

The weight of user behavior evidence is determined by using the formula below:

$$
\begin{gathered}
M_{\mathrm{i}}=\prod_{j=1}^{n} P_{i j} ; \\
W_{i}^{\prime}=\sqrt[n]{M_{i}} ;
\end{gathered}
$$




$$
W_{i}=\frac{W_{i}^{\prime}}{\sum_{i=1}^{n} W_{i}^{\prime}} .
$$

Transforming $W_{i}$ into vector representation is the user behavior evidence weight:

$$
W=\left[\begin{array}{lllll}
W_{1} & W_{2} & W_{3} & \ldots & W_{n}
\end{array}\right]^{T}
$$

\subsection{Calculate the User Behavior Trust Value (UBTV)}

After getting the index weight of the user's behavior evidence, we can use the evidence of membership degree to calculate the user behavior trust value based on per time span. The calculation formula is as follow:

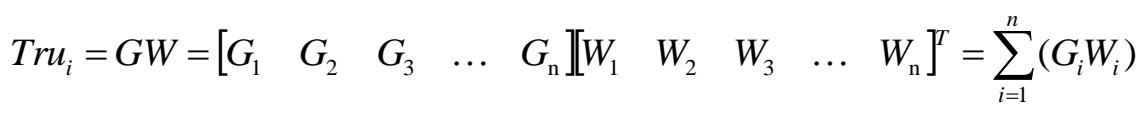

\subsection{Updating Mechanism of UBTV}

According to the trust mechanism of the double sliding window, the user behavior trust records were divided into three types, respectively for User actual trust records, $N_{\min }$ trust records in a small window and $N_{\max }$ trust records in a lager window. The three types of trust records need to be updated to obtain the comprehensive trust value, respectively, using (7), (8) and (9).

$$
\begin{gathered}
\text { Tru_m }=\sum_{i=1}^{m}\left(d_{i} \times \operatorname{Tr} u_{i}\right) \\
T r u_{-} N_{\min }=\sum_{j=1}^{N_{\min }}\left(d_{j} \times \operatorname{Tr} u_{j}\right) \\
T r u_{-} N_{\max }=\sum_{k=1}^{N_{\max }}\left(d_{k} \times \operatorname{Tr} u_{k}\right)
\end{gathered}
$$

\subsection{Comprehensive Calculating of UBTV}

The calculation of user behavior comprehensive trust values is the conservative strategy, thus to take smaller values of the actual trust records comprehensive trust value and the window of all trust records comprehensive trust value. This can not only prevent malicious users from using a few times communication form high trust value of cheating, but also reflect the true value of distrusted users, and the final trust value (Trust) was determined by three of the following discussions:

$$
\begin{gathered}
\text { When } m<N_{\min }: \text { Trust }=\left\{\begin{array}{c}
\text { Tru } u_{-} N_{\min }, T r u_{-} m>T r u_{-} N_{\min } ; \\
\text { Tru } m, T r u_{-} m \leq T r u_{-} N_{\min } .
\end{array}\right. \\
\text { When } N_{\min }<m<N_{\max }: \text { Trust }=T r u_{-} m ; \\
\text { When } m>N_{\max }: \text { Trust }=T r u_{-} N_{\max } ;
\end{gathered}
$$

\section{Simulation Result and Analysis}

\subsection{Simulation Experiment Related Settings}

In order to verify the user behavior evaluation mechanism based on double evidence classification, we used Matlab to conduct a simulated experiment on the computer, where 
the computer hardware configuration and software are as Table 1. The simulation experiment used the $O W L S-T C$ database resources, and set the scenario as a resource sharing and downloading service. The purpose of the user access network is to download the resources. The service providers provide the corresponding service according to the users of different levels of trust.

Table 1. The Computer Hardware and Software Configuration

\begin{tabular}{|c|c|}
\hline CPU & Intel core i7 \\
\hline Clock speed & $2.5 \mathrm{GHz}$ \\
\hline Memory & $8 \mathrm{~GB}$ \\
\hline Software & Matlab R2014a \\
\hline
\end{tabular}

The definition of the user in the experiment is as follows:

Experiment users have passed the authentication of the trusted network, showing that the user's identity is credible, and experiments mainly through differences in behavior of different users are a credible assessment of behavior.

In the experiment, the users were classified into 3 categories according to their quality for, respectively, the High Trusted Users (HTU), Normal Users $(N U)$ and Malicious Users $(M U)$. Different user types correspond to different trust intervals and service categories as shown in Table 2.

Table 2. Trusted Level-Service Mapping Table of Different Users

\begin{tabular}{|c|c|c|l|}
\hline Users & Trust interval & Trust level & \multicolumn{1}{|c|}{ Service level } \\
\hline HTU & {$[0.8,1]$} & High & Refuse or Read-only \\
\hline NU & {$[0.4,0.8)$} & Normal & Allows download and general speed \\
\hline MU & {$[0,0.4)$} & Low & Fast, safety and reliability \\
\hline
\end{tabular}

The resource sharing network is ideal, and the service provider manages the interaction between the users according to the user behavior trust value.

Simulation of user behavior evidence was divided into 30 time span, and the behavior evidence within the time span was divided into twelve species. These twelve species of behavior evidence were classified as: Performance Characteristics $(P C)$, Availability Characteristics $(A C)$ and Safety Characteristics $(S C)$, as shown in Table 3.

Table 3. Characteristic Classification of User Behavior Evidence

\begin{tabular}{|c|c|c|}
\hline Code & Evidence & Classification \\
\hline P1 & IP packet transmission delay(IPTD) & PC \\
\hline P2 & CPU utilization ratio(CPUR) & PC \\
\hline P3 & IP packet throughput(IPT) & PC \\
\hline P4 & IP packet response time(IPRT) & PC \\
\hline A1 & Bit rate error(BRE) & AC \\
\hline A2 & Packet loss rate(PLR) & AC \\
\hline A3 & Connection success rate(CSR) & AC \\
\hline A4 & Trouble free service(TFS) & AC \\
\hline S1 & Illegal connection times(ICT) & SC \\
\hline S2 & Scan port number(SPN) & SC \\
\hline S3 & Ultra vires number(UVN) & SC \\
\hline S4 & Infected viruses number(IVN) & SC \\
\hline
\end{tabular}




\subsection{Accuracy Analysis}

In this simulation experiment, we will use DEC-UB to evaluate the three types of users with different characteristics in the same network environment and interaction mode, and according to the trust value to test the accuracy of DEC-UB. The initial trust value is set to 0.5 .

In theory, the trust value of the three kinds of users will show different trends with the increase of interaction times. HUT behavior trust values will continue to improve, the rising speed is faster, and the trust interval is $[0.8,1]$; NU behavior trust values will gradually increase, but the rate of increase is relatively gentle and the trust interval is $[0.4,0.8)$; MU behavior trust value will continue to decline until the malicious users are removed from the trusted list, and the trust interval is [0.0.4).

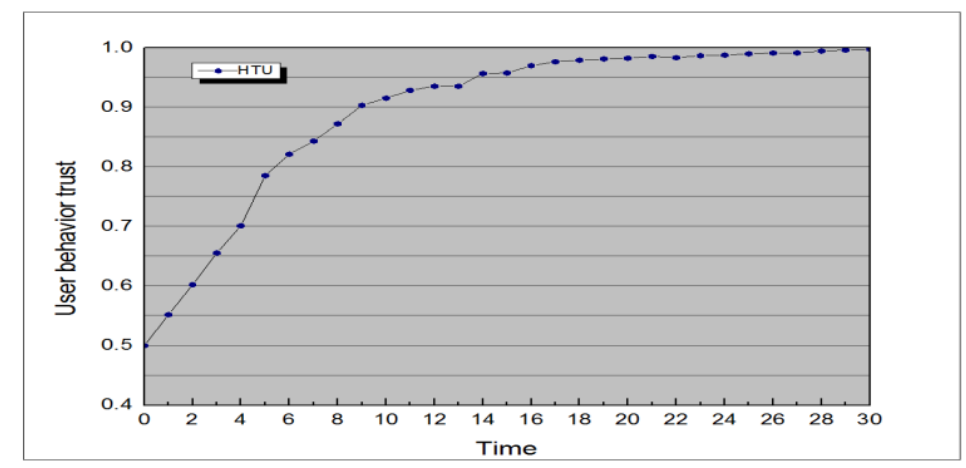

Figure 4. User Behavior Trust Value's Change of HTU

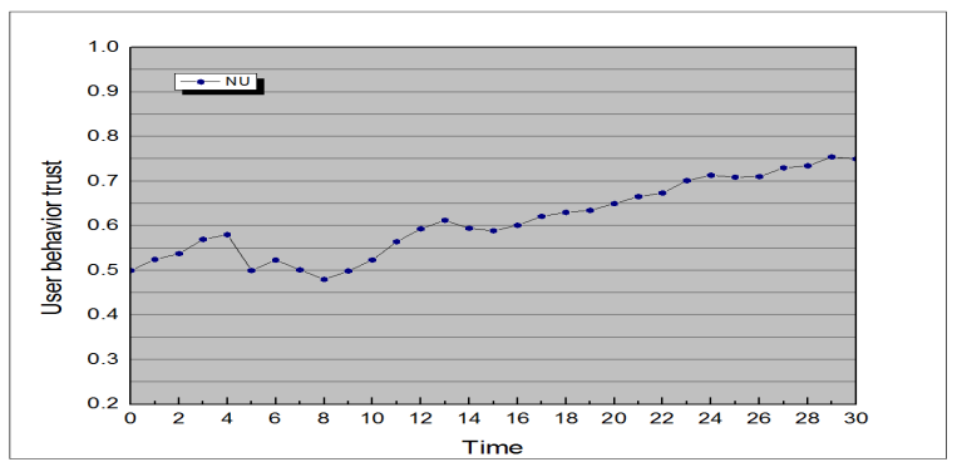

Figure 5. User Behavior Trust Value's Change of NU

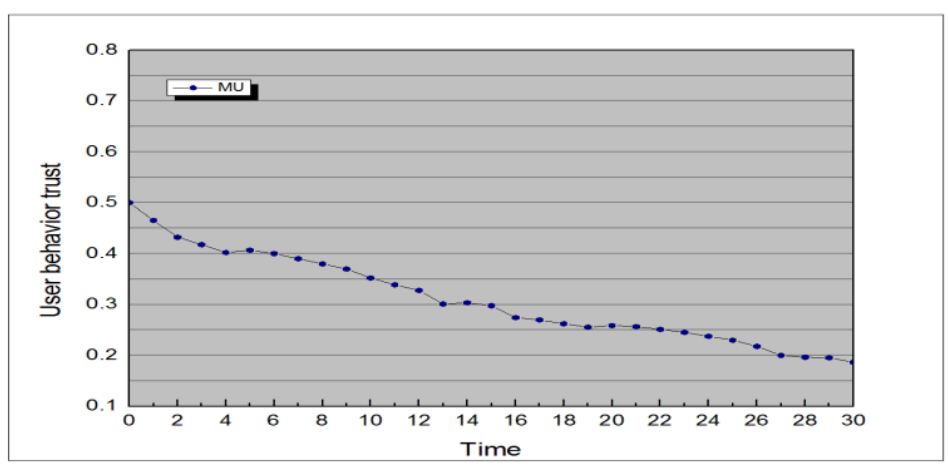

Figure 6. User Behavior Trust Value's Change of MU

As shown in Figures 4-6, the initial trust value was 0.5, and with the increase of interactions, the trust value of HTU rose, and quickly rose to 0.8 after ten times 
evaluation, eventually close to 1 . The trust value of NU tended to be stable, with the increase of interactions, its trust value also gradually increased, but compared to the HTU the overall trend was slowly rising, and there are ups and downs between 0.4 and 0.8 . Because of the existence of fraud, exaggeration, slander or other acts from users, MU's trust value decreased rapidly to 0 with the increase of interactions, and finally was removed from the trusted list. These results verify the accuracy of evaluation based on DEC-UB, and the theoretical analysis is consistent with the experimental results.

\subsection{Comprehensive Analysis}

In order to fully explain the advantages of real-time evaluation mechanism based on DEC-UB, we will compare the DEC-UB with other two user behavior trust evaluation methods. The first one is the evaluation method based on Analytic Hierarchy Process (AHP) [17], the second one is the evaluation method based on Fuzzy Decision [16]. The experimental set of user nodes are 100 in number, where the HTU ratio is 0.3 , the NU ratio is 0.6 , and the MU ratio is 0.1 . The number of user interaction is 200 times, and uses the Successful Transaction Rate (STR) to measure the above three evaluation methods.

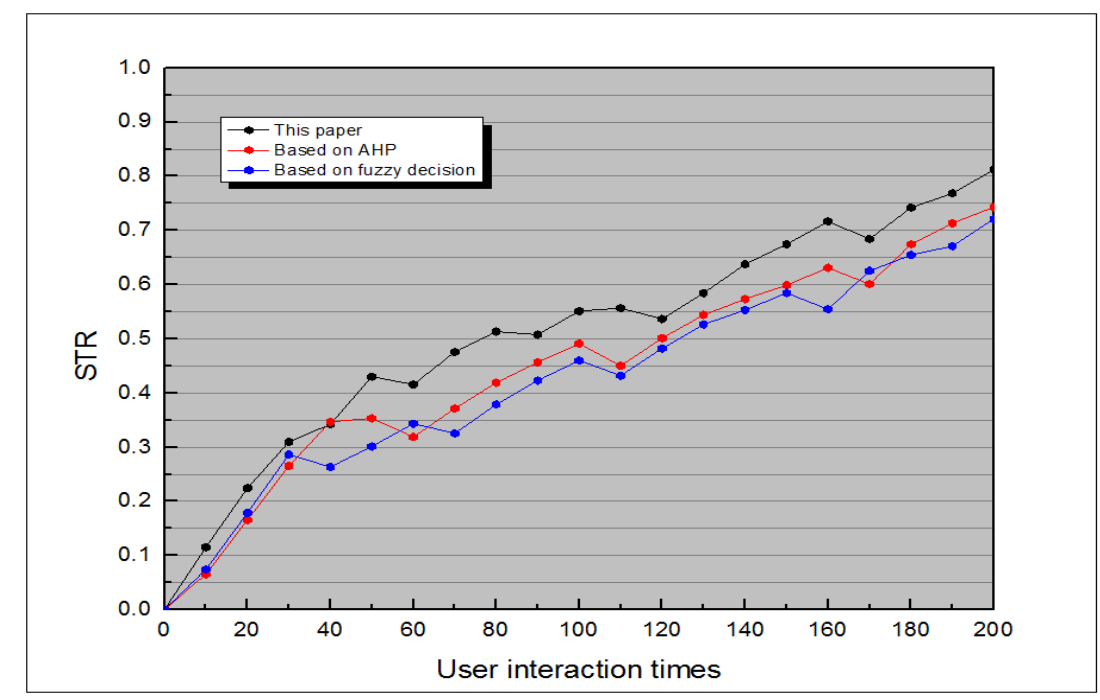

Figure 7. The Authorization Flow Chart Model

As shown in Figure 7, with an increase in the number of interactions the successful transaction rate of all three kinds of trust evaluation methods all showed an upward trend, but the successful transaction rate had a greater volatility in the decision-making process based on AHP evaluation method. The main reason is that the expert opinion plays a key role in the process of obtaining and rating evidence, which means that the standardization of the weight of evidence is subjective, and quantitative results fluctuations are more obvious. The successful transaction rate was low in the decision-making process based on the Fuzzy decision evaluation method, and also had a certain degree of volatility. The main reason for this was that this method of user behavior trust membership rating was not accurate enough, and the weight of evidence was not comprehensive, which led to a coarse-grained trust evaluation result, and left the overall successful transaction rate low.

The proposed evaluation mechanism based on DEC-UB can doubly classify the user behavior evidence, and the double slide window mechanism will allow control the trust evaluation process in real-time, letting the user behavior evidence at any time be directly involved in the trust evaluation, making the evaluation result more comprehensive and accurate. What's more, the trust values are calculated using a simple iterative update method, which makes the evaluation result more quantitative. 


\section{Conclusion}

In this paper, we have analyzed the shortcomings and deficiencies of traditional trusted network user behavior evaluation mechanisms, based on the comprehensive analysis of the characteristics of the terminal environment and the credibility of the user's behavior. We have proposed a real-time evaluation mechanism based on the double evidence classification of user behavior, namely DEC-UB, to evaluate in real-time the user behavior. This evaluation mechanism can double classification of user behavior evidence, and the time factor will control in real-time the trust evaluation process, meaning the user behavior evidence at any time can be directly involved in the trust evaluation, and the evaluation result is more comprehensive and accurate. What's more, the trust values are calculated using a simple iterative update method, which makes the evaluation result more quantitative.

Experiments show that DEC-UB can accurately measure the dynamic change characteristics of the user's behavior, and the evaluation results are reasonable and accurate. In addition it is more practical in terms of safety and accuracy when compared with other models. In future work, we will carry out formal analysis and verification of real-time evaluation mechanism based on DEC-UB, and combine it with the trusted network connection control specification, to create a prototype system that is built to realize the dynamic adjustment from the connection to the authorization.

\section{Acknowledgment}

The work described in this paper is supported by the Tianjin Key Investment Talent Introduction Project Fund (NO: 029416).

\section{References}

[1] Y. Tan, D. Wang, L. H. Fu, and Y. F. Zhao, "Managing behavior trust in networks using statistical methods", Journal of Computation Information Systems, vol. 8, (2012), pp. 4959-4967.

[2] D. Ceolin, P. Groth, A. Nottamkandath, W. Fokkink, and W. R. Hage, "Analyzing User Demographics and User Behavior for Trust Assessment", Lecture Notes in Computer Science (including subseries Lecture Notes in Artificial Intelligence and Lecture Notes in Bioinformatics), vol. 8816, (2014), pp. 219241.

[3] R. Xu, G. Q. Xu, D. Z. Sun, X. H. Li, and Z. Y. Feng, "Improving Keynote Trust Management Model Based On User Behavior For Social Learning", Lecture Notes in Computer Science (including subseries Lecture Notes in Artificial Intelligence and Lecture Notes in Bioinformatics), vol. 8390, (2015), pp. 96103.

[4] A. Mhamed, M. Zerkouk, A. Husseini, B. Messabih and B. Hassan, "Towards a context aware modeling of trust and access control based on the user behavior and capabilities", Lecture Notes in Computer Science (including subseries Lecture Notes in Artificial Intelligence and Lecture Notes in Bioinformatics), vol. 7910, (2013), pp. 69-76.

[5] J. Ma, and Y. S. Zhang, "Research on trusted evaluation method of user behavior based on AHP algorithm", Proceedings of the 2015 7th International Conference on Information Technology in Medicine and Education, (2015), pp. 588-592.

[6] Y. H. Zhong, B. Bhargava, Y. Lu, and P. Angin, "A computational dynamic trust model for user authorization", IEEE Transactions on Dependable and Secure Computing, vol. 12, (2015), pp. 1-15.

[7] T. J. Azderska, "Co-evolving trust mechanisms for catering user behavior", IFIP Advances in Information and Communication Technology, vol. 374, (2012), pp. 1-16.

[8] M. Jaiganesh, M. Aarthi and A. K. A. Vincent, "Fuzzy ART-based user behavior trust in cloud computing", Advances in Intelligent System and Computing, vol. 324, (2015), pp. 341-348.

[9] G. Y. Lin, D. R. Wang, Y. Y. Bie and M. Lei, "MTBAC: A mutual trust based access control model in cloud computing", China Communications, vol. 11, (2014), pp. 154-162.

[10] J. J. Li and L. Q. Tian, "User's behavior trust evaluate algorithm based on cloud model”, Proceedings of the 2015 5th International Conference on Instrumentation and Measurement, Computer, Communication, and Control, (2015), pp. 556-561.

[11] S. B. Hosseini, A. Shojaee and N. Agheli, "A new method for evaluating cloud computing user behavior trust”, Proceedings of the 2015 7th Conference on Information and Knowledge Technology, (2015): 7288735 . 
[12] T. L. Dang, Z. Yan, F. Tong, W. D. Zhang and P. Zhang, "Implementation of trust-behavior based reputation system for mobile applications", Proceedings of the 2014 9th International Conference on Broadband and Wireless Computing, Communication and Applications, (2014), pp. 221-228.

[13] Z. Yan, P. Zhang and R. H. Deng, "TruBeRepec: A trust-behavior-based reputation and recommender system for mobile applications", Personal and Ubiqitous Computing, vol. 16, (2012), pp. 485-506.

[14] M. Kim, and S. O. Park, "Trust management on user behavioral patterns for a mobile cloud computing", Cluster Computing, vol. 16, (2013), pp. 725-731.

[15] G. Hua, M. X. Wu and S. Y. Cui, "A mobile user preference prediction method based on trust and link prediction", Journal of Electronics and Information Technology, vol. 35, (2013), pp. 2971-2977.

[16] J. Y. Ma, Z. J. Zhao and X. Y. Ye, "User Behavior Assessment in Trusted Network Based on Fuzzy Decision Analysis", Computer Engineering, vol. 13, (2011), pp. 125-127+131.

[17] G. T. Ji, L. Q. Tian, Z. X. Hu and J. X. Sun, "AHP-based user behavior evaluation method in trustworthy network", Computer Engineering and Applications, vol. 19, (2007), pp. 123-126_151.

\section{Authors}

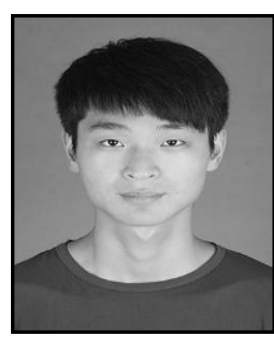

Jiale Zhang, born in 1994. He is a master candidate at the School of Computer Science \& Software Engineering, Tianjin Polytechnic University. His main research interests include trusted computing and network security, etc.

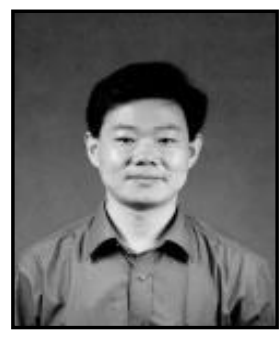

Guiling Zhang, born in 1966. He is a Phd. Professor at the School of Computer Science \& Software Engineering, Tianjin Polytechnic University.. Member of China Computer Federation. His main research interests include network security and image processing, etc.

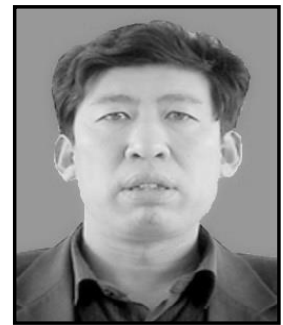

Xiufang Zhang, born in 1963. He is a associate professor at the Institute of Mathematics \& Information Science, Langfang Teachers University. His main research interests include network security, etc. 